\title{
Targeted delivery of a suicide gene to human colorectal tumors by a conditionally replicating vaccinia virus
}

\author{
J Foloppe, J Kintz, N Futin, A Findeli, P Cordier, Y Schlesinger, C Hoffmann, C Tosch, J-M Balloul \\ and P Erbs \\ Transgene SA, 11 rue de Molsheim, Strasbourg Cedex, France
}

\begin{abstract}
We have generated a thymidine kinase gene-deleted vaccinia virus (VV) (Copenhagen strain) that expressed the fusion suicide gene FCU1 derived from the yeast cytosine deaminase and uracil phosphoribosyltransferase genes. Intratumoral inoculation of this thymidine kinase genedeleted VV encoding FCU1 (VV-FCU1) in the presence of systemically administered prodrug 5-fluorocytosine (5-FC) produced statistically significant reductions in the growth of subcutaneous human colon cancer in nude mice compared with thymidine kinase gene-deleted $V V$ treatments or with control 5-fluorouracil alone. A limitation of prodrug therapies has often been the requirement for the direct injection of the virus into relatively large, accessible tumors. Here we
\end{abstract}

demonstrate vector targeting of tumors growing subcutaneously following systemic administration of VV-FCU1. More importantly we also demonstrate that the systemic injection of VV-FCU1 in nude mice bearing orthotopic liver metastasis of a human colon cancer, with concomitant administration of 5-FC, leads to substantial tumor growth retardation. In conclusion, the insertion of the fusion FCU1 suicide gene potentiates the oncolytic efficiency of the thymidine kinase gene-deleted $V V$ and represents a potentially efficient means for gene therapy of distant metastasis from colon and other cancers.

Gene Therapy (2008) 15, 1361-1371; doi:10.1038/gt.2008.82; published online 15 May 2008

Keywords: cancer gene therapy; oncolytic virus; vaccinia virus; suicide gene FCU1

\section{Introduction}

Colorectal cancer is the third most commonly diagnosed malignancy and the second leading cause of cancer mortality in Europe and the United States that accounts for an estimated $10-15 \%$ of newly diagnosed cancer cases and approximately 200000 deaths each year. ${ }^{1,2}$ The liver is the most common site of metastasis, with up to $70 \%$ of colorectal cancer patients eventually developing liver metastases, which are still confined to the liver at the time of metastatic diagnosis in $30-40 \%$ of these patients. However, only one-fourth of patients with metastases confined to the liver are surgical candidates due to tumor size, distribution or accessibility. ${ }^{3,4}$ The mainstay of nonsurgical treatment for advanced colorectal cancer has been 5-fluorouracil (5-FU), and current chemotherapeutic regimens generally consist of 5-FU/leucovorin (folate) combined with newer agents, such as CPT-11 ${ }^{5,6}$ or oxaliplatin. ${ }^{7,8}$ Nonetheless, whereas early-stage, organ-confined disease is associated with a good prognosis, the 5-year survival rate is still extremely poor for advanced colorectal cancer defined by the presence of metastatic disease. Thus, the development of new

Correspondence: Dr P Erbs, Transgene SA, 11 rue de Molsheim, Strasbourg Cedex 67082, France.

E-mail: erbs@transgene.fr

Received 21 December 2007; revised 1 April 2008; accepted 2 April 2008; published online 15 May 2008 therapeutic approaches that can affect liver metastasis represents one of the most important factors for improving the prognosis of patients with advanced colon cancer.

A variety of gene therapy approaches for advanced colorectal cancer have been investigated, in particular suicide gene therapy that results in the intracellular conversion of nontoxic prodrugs into potent chemotoxins directly within the cancer cell. Bacterial and/or yeast cytosine deaminase (CDase) is a well-characterized enzyme-prodrug system that converts the relatively nontoxic anti-fungal agent 5-fluorocytosine (5-FC) to 5-FU. 5-FU is capable of nonfacility diffusion into and out of cells resulting in significant bystander effect of CDase/ 5-FC. ${ }^{9}$ Antitumor effect of CDase/5-FC combination on colon carcinoma was shown both in vitro and in vivo, and clinical trials have been reported showing safety of the CDase/5-FC combination. ${ }^{10}$ However, promising experimental results were not translated into significant curative effect in patients mostly due to low expression of therapeutic transgene bringing only slight therapeutic benefit. ${ }^{11}$

There have been several attempts to increase the efficiency of CDase/5-FC therapy by achieving higher conversion of prodrug 5-FC to 5-FU and the toxic metabolites production. Yeast CDase was shown to deaminate 5-FC more efficiently than bacterial CDase. ${ }^{12,13}$ Moreover, construction of bifunctional fusion gene CDase::uracil phosphoribosyltransferase (CDase:: UPRTase) was reported to shortcut rate-limiting 
enzymatic steps of the 5-FC/5-FU conversion, thus resulting to greatly enhance the sensitivity of the cells to 5-FC compared with CDase alone. ${ }^{14,15}$ Expression of the fusion yeast CDase::UPRTase gene, designated FCU1, has been reported to increase the antitumor effect in experimental animals in vivo. ${ }^{15,16}$ The success of any gene therapy strategy relies on efficient delivery of the transgene specifically to target cells to achieve high therapeutic efficacy whereas limiting unwanted systemic side effects. The use of replication competent viruses is an attractive strategy for tumor therapy because the virus can replicate and spread in situ, exhibiting oncolytic activity through a direct cytopathic effect (CPE). ${ }^{17}$ Currently, viruses such as adenovirus, herpes virus, reovirus and vaccinia virus (VV) are being used as replicating vectors ${ }^{18-21}$ and recent studies using an oncolytic adenovirus shown survival benefit in clinical trials for advanced colorectal cancer. ${ }^{22,23}$ However, there remains a need for more potent agents that will achieve more durable effects and will persist in residual tumor tissues.

Efficient replication, cell lysis and spread of VV, its broad host range along with its remarkable safety record in human use, make VV a very attractive vector for developing oncolytic viruses. ${ }^{21}$ A thymidine kinase (TK) gene-deleted virus has been shown to have decreased pathogenicity compared with wild-type virus, with preserved replication in tumor cells. ${ }^{24}$ Previous studies from our laboratory have shown that TK gene-deleted VV preferentially replicates in tumors in mice when injected intravenously. ${ }^{25}$ To examine whether the FCU1/ 5-FC system could be used with VV as a vector delivery system, we generated a TK gene-deleted VV expressing the yeast fusion FCU1 gene (VV-FCU1). We show here that the expression of the transgene does not detrimentally affect the VV replication and results in the generation of high levels of CDase and UPRTase enzyme activities. We used both subcutaneous (s.c.) and multifocal liver metastases models of LoVo colorectal cancer to test the therapeutic effectiveness of VV-FCU1. Our results indicate that VV-FCU1 delivered by systemic injection can achieve significant growth inhibition in these s.c. and orthotopic models, indicating the potential utility of this vector for advanced colorectal cancer.

\section{Results}

\section{Virus construction and in vitro infection}

The constructed viruses are shown in Figure 1a. The coding sequence of FCU1 and green fluorescent protein $(G F P)$ were introduced into the TK locus under transcriptional control of the synthetic vaccinia promoter p11k7.5, as described in the Materials and methods. Virus structures were confirmed by multiple PCRs.

A series of human cancer cell lines (LoVo, WiDr and A549) were tested for their susceptibility to VV infection in vitro using the VV-GFP. The transduction efficiency was assessed by flow cytometry $14 \mathrm{~h}$ post-infection. These human tumor cell lines showed $\sim 70 \%$ transduction efficiency at a multiplicity of infection (MOI) of 0.1. These results are comparable to those obtained with the modified vaccinia virus Ankara (MVA) strain. ${ }^{16}$ Expression of the FCU1 fusion protein in the vaccinia context was confirmed by western blot using the mouse monoclonal

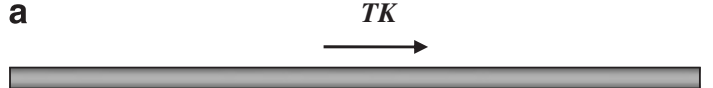

VV
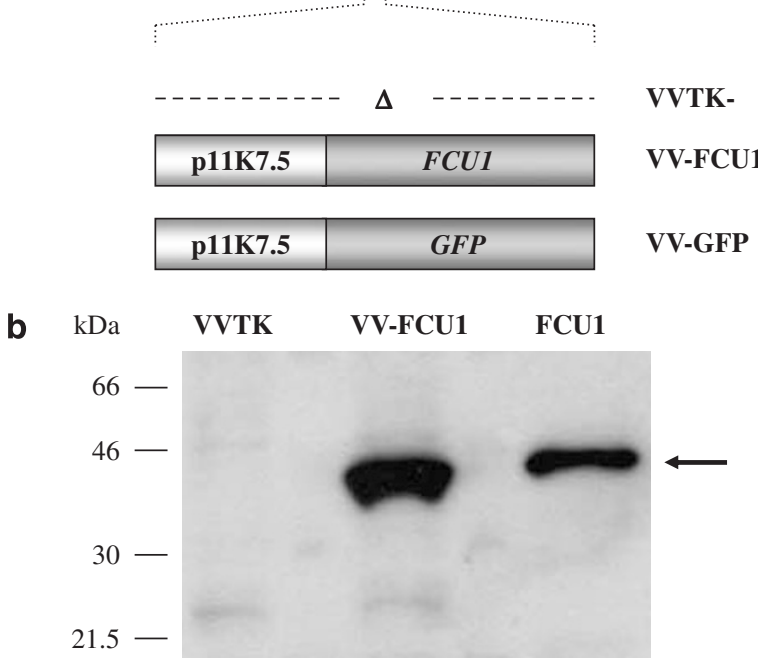

Figure 1 Generation of vaccinia virus (VV) expressing the FCU1 gene and evaluation of the FCU1 protein expression. (a) Schematic representation of viruses used in this study. The VVTK- genome contains a deletion within the thymidine kinase (TK) gene (indicated by $\Delta$ ). VV-FCU1 and VV-green fluorescent protein (GFP) contain in the TK locus the indicated transgenes under the control of the vaccinia synthetic p11K7.5 promoter. (b) Specific detection of the FCU1 protein on western blots by monoclonal antibody (mAb) 3H1. Lane 1 (left to right), LoVo cells infected with VVTK $^{-}$; Lane 2, LoVo cells infected with VV-FCU1; Lane 3, bacterially expressed and purified FCU1 protein. Molecular weight standards are shown in $\mathrm{kDa}$ on the left. The presence of FCU1 $\left(M_{\mathrm{r}}\right.$ 42000 ) is indicated (arrow). Cellular lysates or purified FCU1 protein were loaded at $30 \mu \mathrm{g}$ or $150 \mathrm{ng}$ per lane, respectively.

antibody directed against FCU1 (Figure 1b). Western blot shows that VV-FCU1 expressed the expected 42-kDa FCU1 protein (Figure 1b). Despite the low MOI of infection (0.0001), the level of FCU1 expressed by VV-FCU1 was detectable at $48 \mathrm{~h}$ following infection.

\section{Analysis of the FCU1 enzymatic assays and bystander effect}

Confirmation of expression of functional FCU1 by VVFCU1 was next examined by measuring the enzymatic activities of FCU1 as described previously. ${ }^{16}$ The CDase and UPRTase activities were determined $48 \mathrm{~h}$ postinfection by the analysis of the enzymatic conversions of 5-FC to 5-FU and 5-FU to 5-fluorouridine monophosphate (5-FUMP), respectively. This was determined using lysates prepared from human LoVo tumor cells infected at an MOI of 0.0001 by VVTK $^{-}$and VV-FCU1. This analysis indicates that elevated CDase and UPRTase activities were found in cells infected with VV-FCU1, whereas no CDase and UPRTase activities were detectable in mock-infected cells or in $\mathrm{VVTK}^{-}$-infected cells (Table 1).

A major strength of any prodrug activation model is the potential to extend the cytotoxic therapeutic effect to untransfected cells. In the case of FCU1/5-FC, an efficient bystander effect has been reported as 5-FU can reach neighboring cells by simple diffusion. An analysis of the cell culture supernatant by high performance 
Table 1 Specific CDase and UPRTase activities in LoVo cell line

\begin{tabular}{lcc}
\hline Vector & CDase & UPRTase \\
& $5-F C \rightarrow 5-F U$ & 5-FU $\rightarrow 5-F U M P$ \\
\hline Mock & ND & ND \\
VVTK $^{-}$ & ND & ND \\
VV-FCU1 & $77.87 \pm 5.82$ & $38.02 \pm 2.96$
\end{tabular}

Abbreviations: CDase, cytosine deaminase; 5-FC, 5-fluorocytosine; 5-FU, 5-fluorouracil; 5-FUMP, 5-fluorouridine monophosphate; ND, not detectable; UPRTase, uracil phosphoribosyltransferase; VV, vaccinia virus.

CDase activities are expressed as the number of nmol of 5-FC deaminated per min per $\mathrm{mg}$ of protein and the number of nmol of 5FU phosphorylated per min per $\mathrm{mg}$ of protein, respectively.

UPRTase activities are expressed as the number of nmol of 5-FC deaminated per min per $\mathrm{mg}$ of protein and the number of nmol of 5FU phosphorylated per min per mg of protein, respectively.

The indicated enzymatic activities were measured as described in the Materials and methods section. Each value represents the average of three independent experiments \pm sd

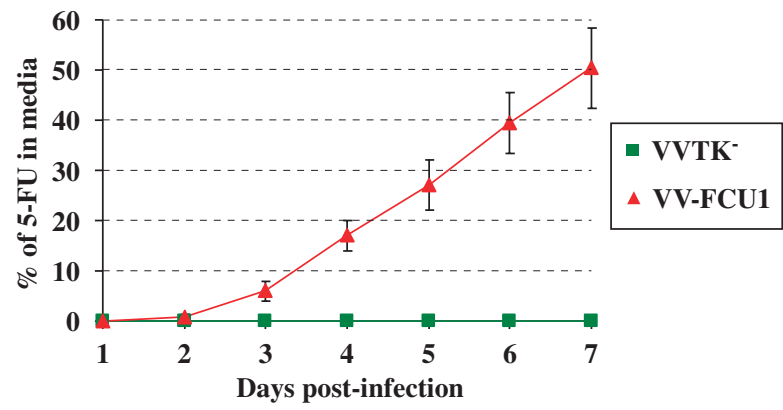

Figure 2 Conversion of 5-fluorocytosine (5-FC) to 5-fluorouracil (5$\mathrm{FU}$ ) and release of 5-FU in the cell culture supernatant. LoVo cells were infected with the indicated vectors at a multiplicity of infection (MOI) of 0.0001 and then incubated with $0.1 \mathrm{mM}$ 5-FC. From days 1 to 7 post-infection, the relative concentrations of 5-FC and 5-FU in the media were measured by high performance liquid chromatography (HPLC). The data are expressed as the percentage of 5-FU in the media relative to the total amount of 5-FC+5-FU. Each data point represents the mean \pm s.d. of triplicate determinations.

liquid chromatography (HPLC) revealed a progressive increase in the amount of 5-FU in the extracellular medium of LoVo cells transduced with VV-FCU1 at an MOI of 0.0001 and incubated with $0.1 \mathrm{mM} 5-\mathrm{FC}$ (Figure 2). This indicates that VV-FCU1-infected cells produce large amounts of FCU1, which are able to catalyze 5-FC into 5-FU that was freely diffusible across cell membranes. In contrast, no 5-FU was detected in the extracellular medium of cells transduced with VVTK(Figure 2). Taken together, our data indicate that VVFCU1 expresses high levels of functional FCU1.

\section{In vitro virus replication in the presence of $5-F C$}

We tested whether the FCU1 gene in combination with 5-FC affects the levels of replication of the VV-FCU1 vector as measured by progeny virus produced. LoVo cells were exposed to $\mathrm{VVTK}^{-}$or VV-FCU1 at an MOI of 0.0001. Infected test cells were either not treated with 5-FC (-) or treated with $0.1 \mathrm{mM} 5-\mathrm{FC}$ immediately (day 0) or $48 \mathrm{~h}$ later (day 2). As shown in Figure 3, there was no effect of the addition of 5-FC to the medium in which cells

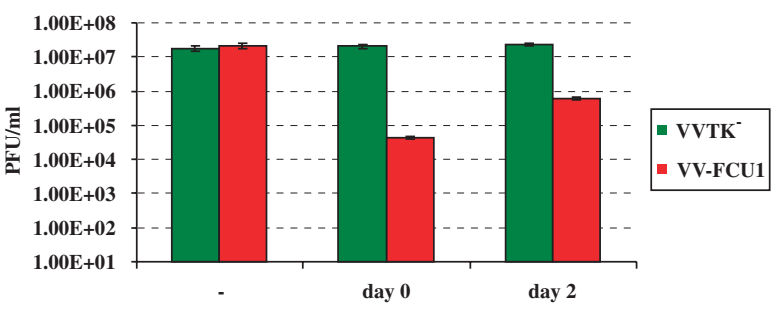

Figure 3 The effect of 5-fluorocytosine (5-FC) on the replication of the vectors. LoVo cells were infected with the indicated vectors at a multiplicity of infection (MOI) of 0.0001 . Cells were not treated with 5 -FC (-) or treated with $0.1 \mathrm{mM} \mathrm{5-FC} \mathrm{immediately} \mathrm{(day} 0$ ) or $48 \mathrm{~h}$ later (day 2) and released virus titers generated after 5 days were determined. Viral titers are shown as plaque-forming unit (PFU) per $\mathrm{ml}$. The results are presented as a mean of triplicate experiments \pm s.d

exposed to the $\mathrm{VVTK}^{-}$virus were cultured. Addition of 5-FC to the cultures of the LoVo cells infected by the VV-FCU1 vector decreases the viral titer by 500 -fold or 35 -fold when the 5-FC was added at 0 or 2 days after infection as compared to cultures to which 5-FC was not added. These results indicated that 5-FC can decrease the level of progeny particles produced by LoVo colon cancer cell line following exposure to the VV-FCU1 vector, and that the inhibition by 5-FC is FCU1 gene-dependent. We conclude that to obtain optimum benefit of viral replication in addition to the suicide gene therapy, addition of prodrug, should be delayed until at least $48 \mathrm{~h}$ following infection.

\section{Cell killing by combination of prodrug activation with viral oncolysis}

We next evaluated the combined oncolytic efficiency of VV-FCU1/5-FC on different cancer cell lines. VV-FCU1 or VVTK $^{-}$was used to infect LoVo, WiDr and A549 cancer cells at an MOI of 0.0001. After $48 \mathrm{~h}, 5-\mathrm{FC}$ was added to the cultures at a range of concentrations, and cell viability was determined 4 days later by trypan blue exclusion. As shown in Figure 4, the oncolytic effect of $\mathrm{VV}$ in the absence of prodrug resulted in $\sim 40-60 \%$ reduction in viable cell number. VVTK ${ }^{-}$and VV-FCU1 in the absence of 5-FC showed no difference in cytotoxicity. The addition of 5-FC did not increase cytotoxicity in tumor cells infected with control $\mathrm{VVTK}^{-}$. In contrast, 5-FC conferred increased toxicity to human tumor cells infected by VV-FCU1 in a prodrug dose-dependent manner (Figure 4). Thus the enhanced cell killing by the combination of VV-FCU1 and 5-FC is due to the expression of FCU1 able to activate the prodrug. In comparison, using the nonpropagative MVA-FCU1 ${ }^{16}$ at an MOI of 0.0001 , no cytotoxicity was observed in presence or absence of 5-FC (data not shown). Despite the inhibitory influence of FCU1/5-FC system on VV propagation, VV-FCU1/5-FC combination therapy was still more effective than either treatment alone.

\section{Biodistribution in mice after systemic vector delivery}

Nude mice bearing established s.c. LoVo tumors were injected intravenously with $1 \times 10^{6}$ plaque-forming unit (PFU) of VV-FCU1. At 14 days after infection of virus, samples of tumor, ovary, brain, lung, lymph nodes, liver, spleen, kidney, colon were harvested, titered on chicken 


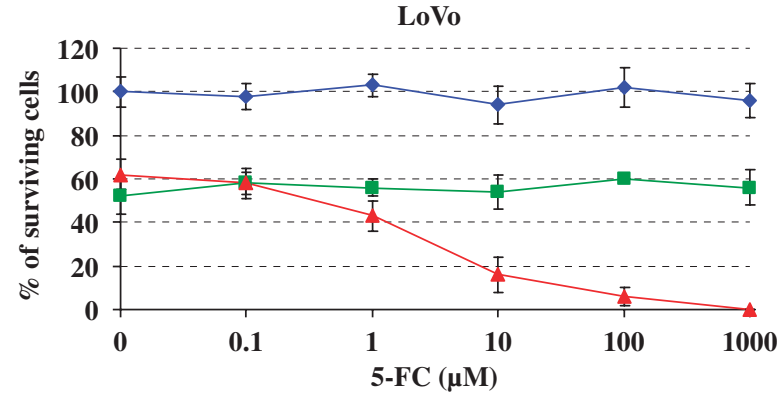

A549

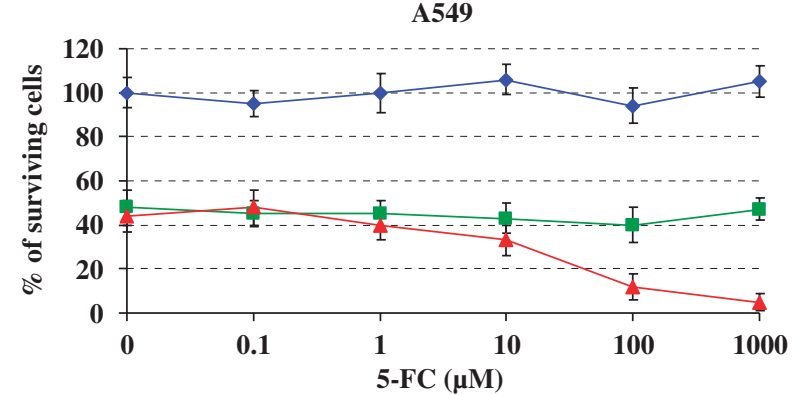

WiDr

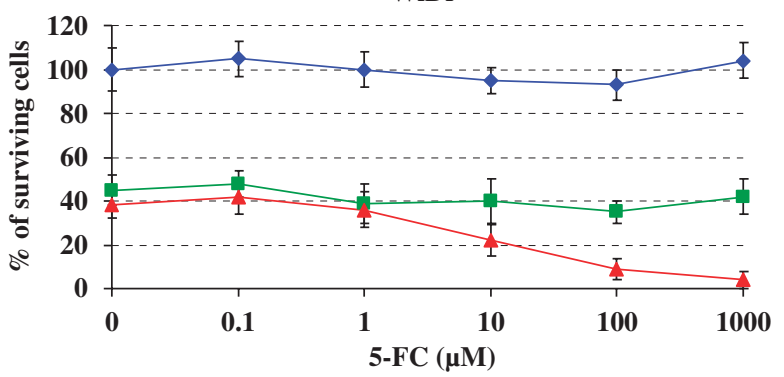

- Mock

- VVTK

\ VV-FCU1

Figure 4 Combination oncolytic and prodrug activation cytotoxicity. Human tumor cells were infected at a multiplicity of infection (MOI) of 0.0001 with the indicated vectors. After $48 \mathrm{~h}, 5$-FC was added at a range of concentration, and cell survival was determined 4 days later as described in Materials and methods section. Results were standardized against values for wells lacking virus and drug, which represented $100 \%$ viability. Values are represented as mean \pm s.d. of four individual determinations.

Table 2 Viral titers of tissues 14 days after infection with VV-FCU1

\begin{tabular}{lccccccccc}
\hline \multicolumn{7}{c}{ Viral titers $\left(\right.$ PFU mg ${ }^{-1}$ tissue $)$} \\
\hline Tumor & Ovary & Brain & Lung & Lymph nodes & Liver & Spleen & Kidney & Colon \\
\hline$(0.2-3.2) \times 10^{5}$ & $2.1-74$ & $0-1.7$ & $0-2$ & 0 & 0 & $0-2.2$ & $0-1.8$ & 0
\end{tabular}

Nude mice bearing established subcutaneous LoVo tumors $(n=3)$ were infected through the tail vein with $1.10^{6}$ PFU of VV-FCU1. On day 14 after infection, tissues were harvested, homogenized and viral titers were determined by a standard plaque assay. The range of virus titers is presented.

embryo fibroblasts (CEF) and viral yield was calculated per milligram of tissue (Table 2). Virus was recovered in high titers from the tumors of all animals. Virus was also detected in ovaries of all three mice but at significantly lower titers. Brain, lung, spleen and kidney were minimally infected (one of three of the mice and with low titer). No detectable infection of the liver, lymph nodes and colon was observed.

\section{In vivo CDase expression following intravenous virus administration}

In vivo Cdase activity was determined after intravenous administration of $1 \times 10^{6}$ PFU of VV-FCU1 into nude mice carrying established s.c. LoVo tumors. Phosphatebuffered saline (PBS) injections were used as negative controls. Groups of mice $(n=3)$ were killed at days 5, 15 and 30 after virus injection. Tumor and tissue samples were harvested and assayed for CDase activity as described. Table 3 shows the results expressed in ng of 5 -FU per mg of protein. CDase activity was detected in all tumors at all time points tested and maximum CDase activity in tumors was observed at day 5 post-injection.
FCU1 expression was still observed in the tumor tissues 30 days post-infection. CDase activity was undetectable in any other tissue tested (ovary, brain, lung, lymph nodes, liver, spleen, kidney and colon) whatever the time point. These findings are consistent with viral recovery results and further suggest tumor-selective gene delivery of VV-FCU1.

\section{$V V$-FCU1/5-FC antitumor activity in a subcutaneous tumor xenograft model}

Nude mice with s.c. LoVo tumors $\left(50-70 \mathrm{~mm}^{3}\right)$ were treated by intratumor or intravenous injections with replicating VV expressing FCU1 or control. 5-FC or control was given per os for 3 weeks starting on day 7 after viral delivery. A single intratumor injection of $1 \times 10^{4} \mathrm{PFU}$ VV resulted in significant inhibition of tumor growth compared with controls $(P<0.01$; Figure 5a). The administration of 5-FC enhanced the antitumor activity of VV-FCU1, when compared to VV-FCU1 without 5-FC $(P<0.05)$, presumably due to the in-situ production of 5-FC derivates through FCU1 gene expression. As expected, the antitumor effect of VV-FCU1 alone was 
Table 3 CDase activity in tumor tissue and healthy organs of nude mice bearing established subcutaneous LoVo tumors $(n=3$ per group time point) after intravenous administration of $1.10^{6} \mathrm{PFU}$ of VV-FCU1

\begin{tabular}{|c|c|c|c|c|c|c|c|c|c|}
\hline \multirow[t]{2}{*}{ Days post-infection } & \multicolumn{9}{|c|}{ CDase activity } \\
\hline & Tumor & Ovary & Brain & Lung & Lymph nodes & Liver & Spleen & Kidney & Colon \\
\hline 5 & $10.36-47.18$ & ND & ND & ND & ND & ND & ND & ND & ND \\
\hline 15 & $5.64-11.69$ & ND & ND & ND & ND & ND & ND & ND & ND \\
\hline 30 & $10.56-12.52$ & ND & ND & ND & ND & ND & ND & ND & ND \\
\hline
\end{tabular}

Abbreviations: CDase, cytosine deaminase; ND, not detectable.

The range of CDase activity is presented. CDase activity is expressed as the number of ng of 5-FU detected per min per mg of protein.

identical to that of $\mathrm{VVTK}^{-}$combined with 5-FC, as VVTK $^{-}$is incapable of 5-FC bioactivation (Figure 5a).

Vaccinia virus given twice intravenously at $1 \times 10^{6}$ PFU showed statistically significant inhibition of tumor growth after 40 days $(P<0.05$; Figure $5 b)$ when compared with vehicle groups. There was no difference between the $\mathrm{VVTK}^{-}$, the $\mathrm{VVTK}^{-}+5-\mathrm{FC}$ and the VV-FCU1 (without 5-FC) groups. As with the intratumor experiment described above, the efficacy of 5-FC administration in combination with VV-FCU1 treatment became more pronounced over time in comparison with VVTKand VV-FCU1 without prodrug ( $P<0.01$; Figure 5b).

\section{VV-FCU1/5-FC antitumor activity in a model of liver metastasis}

In vivo efficacy of VV-FCU1 was next assessed in the orthotopic metastasis model. LoVo tumors were established by splenic injection to form multiple tumor foci in the livers of BALB/c nude mice. At 3 weeks after tumor establishment, a total dose of $1 \times 10^{6}$ PFU VV-FCU1 was injected intravenously and per os 5-FC prodrug treatment was started 10 days after virus administration. After 3 additional weeks, livers from the killed mice were removed and microdissected. Tumor burden is expressed as the percentage of tumor weight per total liver weight (Figure 6). In this experiment, the administration of VVFCU1 in combination with 5 -FC resulted in a reduction in tumor burden compared to that of untreated animals (Figures 6a and b). In contrast, tumor burden was not significantly affected in mice treated with 5-FC or VV-FCU1 alone (Figures 6a and b).

\section{Discussion}

The ideal vector for cancer gene therapy would one which could be delivered through a simple route of administration (for example, intravenous injection), which could access metastatic sites, transduce only the tumor cells, sustain prolonged transgene expression and elicit a systemic anticancer effect.

Thymidine kinase gene-deleted VV has been recently characterized as a selective oncolytic virus showing significant in vitro and in vivo potential as a novel therapy for cancer. ${ }^{26,27}$ The TK-deleted strain has been shown to be dependent on host cell nucleotides and to attenuate the virus by restricting replication to actively dividing cells. $^{24,28,29}$ This TK deletion has been shown to increase viral tumor targeting over other tissues. This was demonstrated in numerous tumor models, including murine colon cancer and melanoma, ${ }^{27}$ murine and human ovarian tumors ${ }^{30}$ and rabbit kidney cancer. ${ }^{31}$ Infection and lysis of $100 \%$ of the cells in a tumor is difficult to achieve in vivo using oncolytic viruses alone. Therefore, oncolytic viruses are often 'armed' with enzyme-prodrug systems which are capable of exerting a strong bystander effect and which may enhance the oncolytic efficacy of the virus therapy by eliminating uninfected tumor cells. The herpes simplex virus TK/ ganciclovir (GCV) has been extensively investigated as an enzyme-prodrug system. The bystander killing effect of that prodrug system has been shown to be mediated by cellular connexins that allow the transfer of the toxic metabolites from the transduced cells to neighboring cells. ${ }^{32,33}$ In contrast to the TK/GCV system; the CDase/ UPRTase/5-FC system may confer a stronger bystander activity that is independent of intercellular junctions. ${ }^{15}$ 5-FC and 5-FU can penetrate tumor cells by passive diffusion and expand the local toxic effect to neighboring cells, irrespective of cellular connexins. Previous studies have demonstrated that yeast CDase deaminates 5-FC more efficiently than Escherichia coli CDase ${ }^{12,13}$ and in our previous report, we noted an increase in the deaminase activity of the fusion protein FCU1 that was over 100-fold higher than the native yeast CDase. ${ }^{15}$

A systemic infusion of the prodrug 5-FC, a Food and Drug Administration-approved antifungal agent, is relatively nontoxic and results in direct intracellular conversion to 5-FU in VV-FCU1-infected tumor cells. ${ }^{16}$ This strategy has the potential to be more effective and less toxic than conventional chemotherapy with systemic 5-FU, and in the clinical setting, could enhance the therapeutic index of combination regimens that currently include 5-FU. Additionally, 5-FC administration may also selectively kill any normal actively dividing cells that might have been inadvertently transduced by the vector expressing FCU1.

In this report, we describe experiments which have assessed the antitumor potential of the recently characterized FCU1 fusion suicide gene ${ }^{15}$ in combination with the oncolytic TK gene-deleted VV as viral vector. We first tested a series of human cancer cell lines for transduction efficiency. Our findings demonstrate that a TK gene-deleted VV can efficiently transduce human tumor cells in vitro, allowing $\sim 70 \%$ gene transfer efficiency at an MOI of 0.1 and $14 \mathrm{~h}$ post-infection. Similar gene transfer efficiencies were obtained with the highly attenuated VV MVA strain. ${ }^{16}$ At the MOI of 0.1 , in contrast to MVA, cells infected by TK gene-deleted VV had severe CPEs $48 \mathrm{~h}$ post-infection (data not shown). 

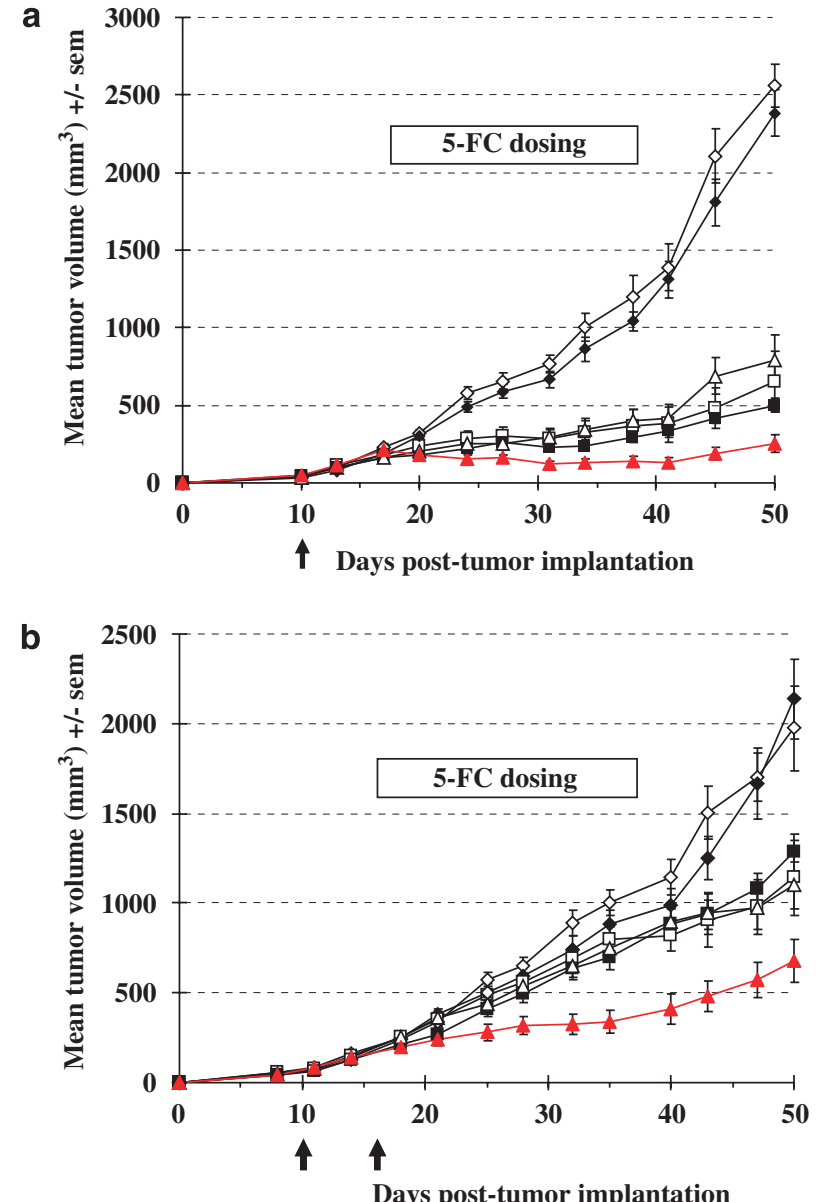

Figure 5 Growth suppression of human colorectal LoVo tumors implanted subcutaneously after intratumoral or intravenous injections of vaccinia virus (VV)-FCU1 plus 5-fluorocytosine (5-FC). (a) Mean tumor volume after intratumoral treatment with $1 \times 10^{4}$ plaque-forming unit (PFU) of replicating VV (indicated by arrow). LoVo cells implanted subcutaneous into nude mice were injected at day 10 with vehicle alone $(\diamond$, vehicle+water; $\diamond$, vehicle+5-FC) or with the indicated virus $\left(\square\right.$, VVTK $^{-}+$water; $\mathbf{\square}, \mathrm{VVTK}^{-}+5-\mathrm{FC}$; $\triangle$, VV-FCU1+water; $\boldsymbol{\Delta}$, VV-FCU1+5-FC). The animals were then treated twice daily from days 17 to 38 with per os administrations of water or 5 -FC ( $200 \mathrm{mg} \mathrm{kg}^{-1}$ per day). The data represent the mean of 13 animals. (b) Mean tumor volume after systemic treatment with $1 \times 10^{6} \mathrm{PFU}$ of replicating VV (indicated by arrows). Mice bearing LoVo subcutaneous xenografts were treated with two intravenous administrations (by tail vein) at days 10 and 17 with vehicle alone $(\diamond$, vehicle+water; $\diamond$, vehicle $+5-\mathrm{FC})$ or with the indicated virus $\left(\square, \mathrm{VVTK}^{-}+\right.$water; $\square, \mathrm{VVTK}^{-}+5-\mathrm{FC} ; \triangle$, VVFCU1+water; $\boldsymbol{\Delta}$, VV-FCU1+5-FC). The animals were then treated twice daily from days 17 to 38 with per os administrations of water or 5-FC ( $200 \mathrm{mg} \mathrm{kg}^{-1}$ per day). The data represent the mean of 14 animals.

In this study, we have shown that a TK gene-deleted VV expressing the yeast CDase/UPRTase fusion gene has potent antitumor effects in the presence of 5-FC, both in vitro in many human tumor lines and in vivo in a murine model of a human colon tumor. In vitro experiments showed that this oncolytic VV expresses high levels of FCU1 as assessed by protein level and enzymatic assays. CDase and UPRTase activities were found to be 10-fold higher in human colorectal tumor cells infected by VVFCU1 at an MOI of 0.0001 than in cells infected by the attenuated VV MVA-FCU1 at an MOI as high as 0.01.16
In addition, our results indicate that VV-FCU1+5-FC generates a strong bystander effect. The observed increase in in vitro cytotoxicity induced by the addition of 5-FC to the VV-FCU1 at low MOI may represent an important advantage in the in vivo therapeutic setting. The chemotherapeutic drug could conceivably limit viral replication and subsequent propagation in the tumor. ${ }^{34,35}$ In vitro, we have shown that the expression of the FCU1 gene plus 5-FC can reduce the viral progeny yield of VVFCU1 vector from the infected colon tumor cells. The 5-FU produced appeared to have an inhibitory effect on viral replication, either by direct interference with viral DNA synthesis or by killing surrounding cells prior to their infection. However, our data show a clear benefit in combining the oncolytic virotherapy using VV-FCU1 and the prodrug 5-FC. Theoretically, prodrug activation might even enhance spread of the oncolytic virus, if direct and bystander cell killing allowed more efficient release and dissemination of progeny virus, even if their number were reduced. Some studies have shown that combined virus/prodrug treatment may enhance the replication/spread of oncolytic adenoviruses in mouse tumor models. ${ }^{36,37}$ A recent study indicated that low doses of 5-FU potentiated herpes simplex virus cytotoxic effects primarily through increasing viral replication by 19-fold. ${ }^{38}$ Clearly, the timing of prodrug administration will be a key factor in the overall efficacy; administration too early in the course of vaccinia infection could be counterproductive, preventing virus replication and thus limiting both the oncolytic effect, and the amount and distribution of the prodrug-activating enzyme. However, with optimum timing, prodrug activation is expected to kill more cells, or do so more rapidly although the tumor is smaller, than could be achieved by viral oncolysis alone.

In the study described in this report, we have demonstrated the ability of intravenously administered TK gene-deleted VV to preferentially target human colorectal tumor cells in mice. Our findings are consistent with previous studies that demonstrate preferential infection of tumors over normal tissues by TK genedeleted VV. For example, Puhlmann et al. ${ }^{27}$ showed that TK gene-deleted VV administered intraperitoneally (i.p.) preferentially infected i.p. tumors compared with healthy vital organs, including lung, liver, heart and kidney. Futhermore, intravenous administration of the luciferase-expressing recombinant VV led to significantly higher luciferase activity in different tumor models compared to normal tissues assayed. ${ }^{27,31}$ In our biodistribution studies, viral recovery from mouse tissues demonstrated that VV-FCU1 was consistently detected in all s.c. tumors whereas other organs remain much less infected. Our measurement of viral infection of organs is done 14 days after injection. It cannot be excluded that infection of nontumor organs, such as ovaries, is the result of secondary infection by virus produced in the tumor. Most organs were negative for VV-FCU1 (liver, colon and lymph nodes) or were minimally infected (one out of three brains, lungs, spleens and kidneys). Only the ovary was shown to be also infected by VV-FCU1 (three out of three ovaries) but with a 4-log lower titer compared with tumors. The infection of murine ovaries by VV was demonstrated previously. 27,34,39,40 Similar tropism of VV was also demonstrated after intravenous administration in rhesus macaques. ${ }^{41}$ We postulate that 
a

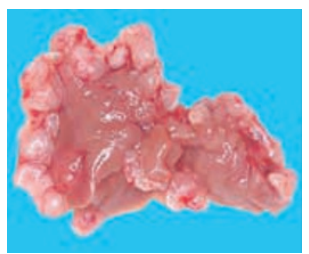

Vehicle

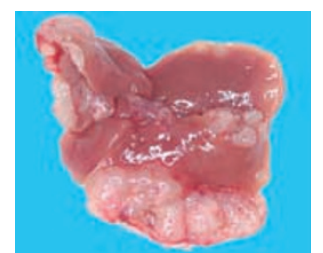

Vehicle + 5-FC

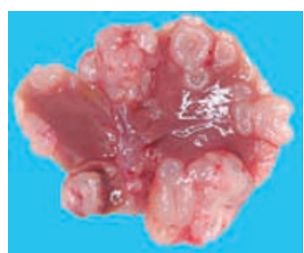

VV-FCU1

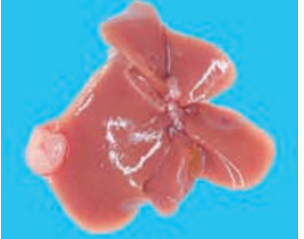

VV-FCU1 + 5-FC

b

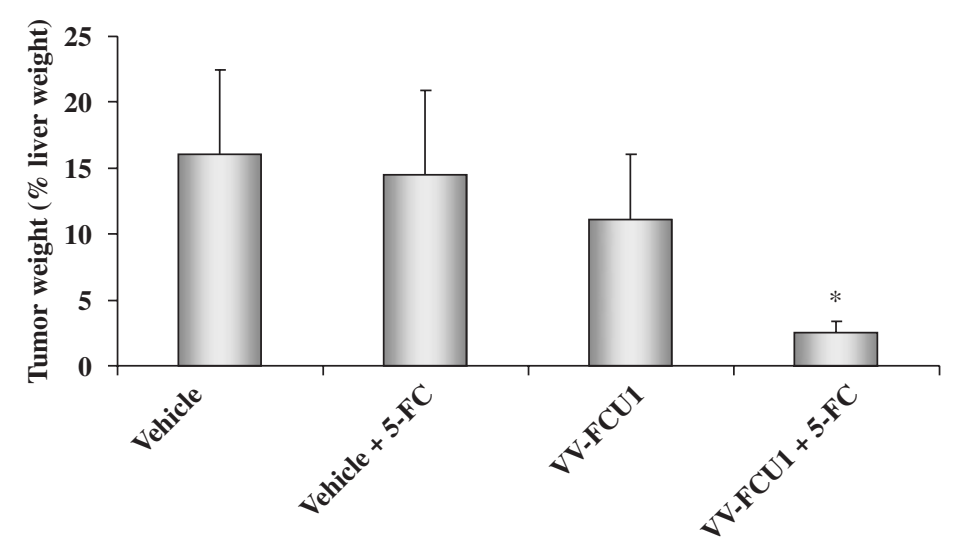

Figure 6 Effect of systemic treatment with vaccinia virus (VV)-FCU1+5-fluorocytosine (5-FC) on orthotopic liver metastasis model. Liver weight of mice bearing liver metastases after one intravenous administration of VV-FCU1 plus 5-FC treatment. At 3 weeks after cell inoculation, $1 \times 10^{6}$ plaque-forming unit (PFU) of VV-FCU1 was injected through the tail vein. 5 -FC administration (200 mg kg ${ }^{-1}$ per day) started 10 days after virus injection and lasted for 3 weeks. Livers were collected and weighted at the end of 5-FC treatment. (a) Macroscopic aspects of characteristic livers. (b) Quantitative analysis of burden of LoVo-derived metastases in liver. Tumor tissues were isolated from the liver tissue by microdissection under a microscope and the total liver weight and tumor weight were measured. The tumor burden is expressed as a percentage of tumor weight to the total liver weight \pm s.e.m. ${ }^{*} P<0.01$ versus vehicle alone. The data represent the mean of eight animals.

the leaky vasculature and dividing cells within the follicle represent a prime site for vaccinia replication. ${ }^{42}$ In this study, we have also evaluated the tissue distribution of VV-FCU1 by measuring the conversion of 5-FC to 5FU. We demonstrated that VV-FCU1 expresses active CDase that converts 5-FC to 5-FU efficiently and preferentially in tumors.

In a s.c. human colon tumor model, after intratumor or intravenous administration of VV-FCU1, we unequivocally demonstrated that VV-FCU1/5-FC produced a superior antitumor effect than either agent acting alone. In a previous study, systemic 5-FU administration in the same s.c. LoVo model under conditions similar to those used in the present study was reported to show no therapeutic efficacy compared with control mice despite the administration of doses of 5-FU that were at the maximum tolerated concentrations (i.p. injection of $10 \mathrm{mg}$ of 5 -FU per $\mathrm{kg}$ twice daily during 2 weeks). ${ }^{15}$

Furthermore, we have demonstrated an appreciable antitumor effect of VV-FCU1 on the liver metastases of a human colon cancer by intravenous injection of $\mathrm{VV}$ FCU1 plus 5-FC treatment.

No clinical signs of disease (death, weight loss, lethargy and hyperactivity) were observed following intravenous inoculation of TK gene-deleted VV Copenhagen strain expressing FCU1 at the dose of $1 \times 10^{6}$ PFU. However, between 5 and 7 days post-injection, mice showed typical cutaneous pox lesions on the tail and footpad (data not shown). This is characteristic of $\mathrm{VV}^{43}$ and the necrotic skin lesion healed slowly and completely over the course of 6 weeks.
To our knowledge, this is the first report to assess the therapeutic efficacy of the Copenhagen strain of VV 'armed' with an enzyme-prodrug system. Previous studies have used the much more pathogenic Western Reserve (WR) strain as an oncolytic VV vector and showed that it selectively replicates in cancer cells. ${ }^{27,34,40}$ Recombinants of the Copenhagen strain of VV have been shown to be 5000 times less virulent than the strain WR when injected intracranially into 3-week-old mice and it is better tolerated by nude mice. ${ }^{43}$ Furthermore, the vaccine Copenhagen strain was approved for human vaccine use in Denmark and the Netherlands. ${ }^{44} \mathrm{~A}$ recombinant of the Copenhagen strain expressing the rabies virus glycoprotein was constructed by the insertion of the encoding cDNA in the TK locus. ${ }^{45}$ This recombinant has received a conditional commercial license in both Europe and in the United States. The recombinant is administered as a live vaccine in baits for oral uptake by foxes in Europe and by raccoons and coyotes in the United States. Extensive seeding of large geographic regions has provided field safety and efficacy data. ${ }^{46}$ Previously, TK gene-deleted VV Copenhagen strain expressing cytokines has been shown to reduce tumor growth after intravenous delivery in immunocompetent mice bearing s.c. murine tumors as well as nude mice bearing human colorectal tumors. ${ }^{25}$ In summary, we have demonstrated that the combination of the tumor-selective strain of the Copenhagen VV and the FCU1/5-FC system is a potent vector for cancer gene therapy. We have shown that systemic injection of VVFCU1 leads to high gene expression within tumor cells 
in vivo. Furthermore, we have shown that intravenous injection of VV-FCU1 followed by systemic 5-FC administration results in a significant antitumor effect in an in vivo model of s.c. and established liver metastases of human colon cancer. Thus, our data provide a strong rationale for the clinical translation of VV-FCU1 as an oncolytic viral vector for the control of colon cancer. Future studies will be performed to optimize these results, including optimization of vector and prodrug delivery and administration modalities. In addition, we are working at improving the safety profile of this virus further by mutating other viral genes. These studies may contribute to the translation of the VV-FCU1/5-FC system into clinical practice.

\section{Materials and methods}

\section{Cell culture}

Human colon cancer cell line WiDr and LoVo, human lung cancer cell line A549, human osteosarcoma cell line 143B (a TK- cell line) and mouse myeloma line Sp2/0Ag14 were obtained from the ATCC (Manassas, VA, USA). All cell lines were grown in Dulbecco's modified Eagle's medium (DMEM) supplemented with $10 \%$ fetal bovine serum. Before use, all cell lines were tested and found to be free of Mycoplasma infection. Primary CEF were used for recombination and amplification of viral vectors. CEF cells were prepared from chicken embryo obtained from fertilized eggs (Charles River SPAFAS, Germany) previously incubated 11 days at $37^{\circ} \mathrm{C}$ in a humid atmosphere. Chicken embryos were dissected and treated with a solution of trypsin $2.5 \%$ (w/v). CEF cells were maintained in Eagle-based medium supplemented with $5 \%$ fetal bovine serum.

\section{Construction and transduction of vaccinia virus}

All recombinant VVs are derivatives of Copenhagen strain. The VV shuttle plasmid contains the vaccinia synthetic early/late p11K7.5 promoter, $^{16}$ surrounded by portions of the vaccinia TK gene, which allows homologous recombination into this locus. From plasmid pRS306-FCU1, ${ }^{15}$ the fragment NotI-KpnI-containing FCU1 gene was cloned into the corresponding sites of the VV shuttle plasmid generating a recombinant shuttle plasmid in which FCU1 is under the control of the vaccinia synthetic early/late p11K7.5 promoter. The generation of recombinant VVs was previously described in detail. ${ }^{45}$ Briefly, CEF cells were infected with VVCopenhagen ts7 (at an MOI of 0.1), incubated at the permissive temperature $\left(33^{\circ} \mathrm{C}\right)$ for $2 \mathrm{~h}$, then transfected with a $\mathrm{CaCl}_{2}$ coprecipitate of $\mathrm{VV}$-Copenhagen wild-type DNA $(1 \mu \mathrm{g})$ and the recombinant shuttle plasmid $(0.2 \mu \mathrm{g})$. The cells were incubated for $48 \mathrm{~h}$ at $39.5^{\circ} \mathrm{C}$. Dilutions of $\mathrm{ts}^{+}$virus emerging were then used to infect the TK-deficient 143B cells in selection medium containing 5-bromo-2'-deoxyuridine at final concentration of $150 \mu \mathrm{g} \mathrm{ml}^{-1}$ (Sigma, St Louis, MO, USA). Positive $\mathrm{TK}^{-}$ plaques were isolated and selected for a second cycle in $143 \mathrm{~B}$ cells in presence of 5-bromo-2'-deoxyuridine. The same methods were used to generate the VV-GFP that expresses GFP under the control of the p11K7.5 promoter. The control VV vector was TK gene-deficient but has no insert $\left(\mathrm{VVTK}^{-}\right)$. Final recombinant VV viruses were amplified in CEF and virus stocks were titrated on CEF by plaque assay.

\section{Enzyme purification}

FCU1 protein was purified in E. coli using the glutathione $S$-transferase (GST) fusion protein system. ${ }^{47}$ The FCU1 gene was cloned into the fusion-protein expression vector pGEX-2T (GE Healthcare, Orsay, France) directly after the GST gene. To isolate the GST:FCU1 fusion protein the supernatant of lysed transformed E. coli BL21 cells was incubated with glutathione-Sepharose $4 \mathrm{~B}$ beads as recommended by the manufacturer (GE Healthcare). Subsequently, thrombin (GE Healthcare) was added to the beads to cleave off the FCU1 protein. The supernatant was then incubated with benzamidine Sepharose beads (GE Healthcare) to remove the thrombin.

\section{Mouse monoclonal antibody generation}

B6D2 mice (Charles River, Saint Aubin-les-Elbeuf, France) were immunized three times s.c. with $10 \mu \mathrm{g}$ of purified FCU1 protein in complete Freud's adjuvant (Sigma) in 2-week intervals followed 4 weeks later by one i.p. boost. Spleen cells from immunized mice were fused 3 days after the last boost with Sp2/0-Ag14 mouse myeloma cells ${ }^{48}$ and plated on $100 \mathrm{~mm}$ Petri plates containing the hybridoma selection medium (ClonaCellHY, StemCell Technologies, Grenoble, France). For detection of specific immunoglobulin (Ig), 499 hybridomas were screened by enzyme-linked immunosorbent assay (ELISA). Three hybridomas supernatant specifically recognized the purified FCU1 protein by ELISA and western blotting. After subcloning these hybridomas by limiting dilution, the three mouse monoclonal antibodies directed against FCU1 (mAb 3H1, mAb 8E1 and mAb 6A6) were purified from hybridoma supernatant by Protein A-Sepharose chromatography (GE Healthcare).

\section{Western blot analysis}

LoVo tumor cells were infected by VVTK ${ }^{-}$and VV-FCU1 at an MOI of 0.0001 and incubated for $48 \mathrm{~h}$. Cell lysate proteins $(30 \mu \mathrm{g})$ (determined using a Bio-Rad protein assay) and $150 \mathrm{ng}$ of purified FCU1 protein were run on a $10 \%$ SDS-polyacrylamide gel electrophoresis (PAGE) gel under reducing conditions and transferred onto a nitrocellulose membrane. The membrane was incubated with mouse monoclonal antibody mAb $3 \mathrm{H} 1\left(1 \mu \mathrm{g} \mathrm{ml}^{-1}\right)$, washed and incubated with secondary antibody coupled horseradish peroxidase (Amersham, Les Ulis, France). Signal detection was done by enhanced chemiluminescence (Amersham).

\section{Enzymatic assays}

CDase activity and uracil phosphoribosyltransferase (UPRTase) activity in LoVo cells were determined using 5-FC (Toronto Research Chemicals Inc., North York, ON, Canada) and 5-FU (Sigma) as substrates. LoVo human tumor cells $\left(3 \times 10^{6}\right.$ cells) were infected by $\mathrm{VVTK}^{-}$and VV-FCU1 at an MOI of 0.0001. At 48 h later, enzymatic assays in cell lysate were determined as previously described. ${ }^{16}$ 5-FC, 5-FU and 5-FUMP were separated isocratically using HPLC (supelcosil LC-18-S column and UV detection at 260 and $280 \mathrm{~nm}$ ). For CDase activity, the mobile phase was $50 \mathrm{~mm}$ phosphoric acid adjust to $\mathrm{pH}$ 2.1 with ammonium hydroxide. For UPRTase activity, the 
mobile phase was $20 \mathrm{mM} \mathrm{KH_{2 }} \mathrm{PO}_{4}, 5 \mathrm{mM}$ tetrabutylammonium sulfate, $5 \%$ methanol adjusted to $\mathrm{pH} 5$ with potassium hydroxyde.

CDase activity was also measured indirectly by measuring 5-FU released in the culture media. LoVo cells were infected with the different vectors at an MOI of 0.0001 and plated in 12-well culture dish $\left(1 \times 10^{6}\right.$ cells per well). After $24 \mathrm{~h}, 0.1 \mathrm{mM} 5$-FC was added to the cultures. At different time points, the concentrations of 5-FC and 5 -FU in the media were measured using HPLC. Media $(50 \mu \mathrm{l})$ were quenched with $1 \mathrm{ml}$ of ethyl acetate/ 2-propanol/0.5 M acetic acid solution (84:15:1). The samples were vortexed and centrifuged. The organic supernatant was evaporated to dryness under a stream of nitrogen at $60{ }^{\circ} \mathrm{C}$ and reconstituted in $50 \mu \mathrm{l}$ of water and analyzed by HPLC using a mobile phase of $50 \mathrm{mM}$ phosphoric acid adjusted to $\mathrm{pH}$ 2.1. The data are expressed as the percent of 5-FU in the media for various incubation times with 5-FC.

\section{In vitro viral growth assay}

Human LoVo cells were transduced in suspension by $\mathrm{VVTK}^{-}$and VV-FCU1 at an MOI of 0.0001. A total of $3 \times 10^{5}$ cells per well were plated in six-well culture dishes in $2 \mathrm{ml}$ of medium. Cells were treated with $0.1 \mathrm{mM}$ 5-FC immediately (at day 0), $48 \mathrm{~h}$ later (at day 2) or never treated with 5-FC. Supernatants and cells were collected at day 5 and after a quick freeze-thaw cycle to release intracellular viral particles viral progeny were quantified on $\mathrm{CEF}$ by plaque assay.

\section{In vitro cell sensitivity to $5-F C$}

Human tumor cells were transduced in suspension by the respective recombinant $\mathrm{VV}$ at an MOI of 0.0001 . A total of $3 \times 10^{5}$ cells per well were plated in six-well culture dishes in $2 \mathrm{ml}$ of medium. At $48 \mathrm{~h}$ after infection, cells were exposed to various concentrations of 5-FC for 4 days, before determination of cell viability by trypan blue exclusion.

\section{Subcutaneous tumor model}

Female NMRI nude mice were obtained from Charles River Laboratories. Animals used in the studies were uniform in age (6 weeks) and body weights ranged from 23 to $26 \mathrm{~g}$. NMRI nude mice were injected s.c. into the flank with $5 \times 10^{6}$ LoVo cells. When tumors reached a diameter of $50-70 \mathrm{~mm}^{3}$, the mice were randomized in a blinded manner and treated with the indicated vectors for the in vivo experiments.

\section{Biodistribution of the virus}

The presence of VV-FCU1 was evaluated by virus titration and by CDase expression in tumors and organ samples. $1 \times 10^{6}$ PFU of VV-FCU1 was injected intravenously by tail vein injection into nude mice bearing established s.c. LoVo tumors. Mice were killed at indicated time points, and the tumors and other organs were collected and weighted. For viral titer determinations, tumors and organs were homogenized in PBS and titers were determined on CEF by plaque assay. Viral titers were standardized to milligram of tissue. The CDase activity in tumors and organs samples after intravenous administration of $1 \times 10^{6}$ PFU of VV-FCU1 was determined using radiochemical 5-FC. Tumors and organ samples were lysed using a polytron in $400 \mu \mathrm{l}$ of lysis buffer (50 mM Tris- $\mathrm{HCl} \mathrm{pH} 7.5,150 \mathrm{mM} \mathrm{NaCl}, 5 \mathrm{mM}$ EDTA, $1 \mathrm{mM}$ dithiothreitol, $1 \%$ Triton $\mathrm{X}-100)$, followed by three freeze-thaw cycles. After centrifugation, $80 \mu \mathrm{l}$ of tissue lysate were incubated with $0.1 \mathrm{mM}\left[6-{ }^{3} \mathrm{H}\right] 5-\mathrm{FC}$ $\left(0.5 \mathrm{nCi} \mu \mathrm{l}^{-1}\right.$, Sigma $)$ for $1 \mathrm{~h}$ at $37^{\circ} \mathrm{C}$. The reaction mixture was analyzed by HPLC as described above using a mobile phase of $50 \mathrm{~mm}$ phosphoric acid adjusted to $\mathrm{pH}$ 2.1. The produced $\left[6-{ }^{3} \mathrm{H}\right] 5-\mathrm{FU}$ was quantified by a online RamonaStar radioactive flow detector (Raytest, Courbevoie, France).

\section{Antitumor activity of VV-FCU1 in subcutaneous tumor model}

Nude mice bearing established s.c. LoVo tumors (50$70 \mathrm{~mm}^{3}$ ) were treated one time intratumorally or two times intravenously (by tail vein) with the indicated vectors at dose of $1 \times 10^{4}$ or $1 \times 10^{6} \mathrm{PFU}$, respectively. Starting day 7 following viral injection, 5-FC was given by oral gavage at $100 \mathrm{mg} \mathrm{kg}^{-1}(0.5 \mathrm{ml} 5$-FC $0.5 \%$ in water) twice a day for 3 weeks. Tumor size was measured twice weekly using calipers. Tumor volume was calculated in $\mathrm{mm}^{3}$ using the formula $(\pi / 6)$ (length $\times$ width $^{2}$ ).

\section{Antitumor activity of VV-FCU1 in an orthotopic liver metastasis model}

A xenogeneic mouse model of human colorectal cancer metastasis to the liver was also established as previously described $^{49}$ by infusion of tumor cells into the portal system through intrasplenic injection. Briefly, $2.10^{6} \mathrm{LoVo}$ cells in $30 \mu \mathrm{l}$ PBS were implanted into the spleens of anesthetized and laparotomized BALB/c nude mice (female, 8 weeks old, Charles River Laboratories). At 3 weeks after tumor cell inoculation, mice were treated one time intravenously (by tail vein) with the indicated vectors at dose of $1 \times 10^{6} \mathrm{PFU}$. At 10 days after viral injection, 5 -FC was given by oral gavage at $100 \mathrm{mg} \mathrm{kg}^{-1}$ $(0.5 \mathrm{ml} 5-\mathrm{FC} 0.5 \%$ in water) twice a day for 3 weeks. Mice were killed at the end of 5-FC treatment and tumorbearing livers were microdissected and weighed. Tumor burden was expressed as the ratio of tumor per total liver weight, as described previously. ${ }^{50}$

\section{Statistical analysis}

Statistical analyses were performed using the nonparametric Mann-Whitney $U$-test and STATISTICA 7.1 software (StatSoft, Maisons-Alfort, France). A $P<0.05$ was considered to be statistically significant.

\section{Acknowledgements}

We thank D Villeval for excellent technical assistance. We acknowledge the help and scientific criticisms of B. Acres during the preparation of this paper.

\section{References}

1 Midgley R, Kerr D. Colorectal cancer. Lancet 1999; 353: 391-399.

2 Jemal A, Siegel R, Ward E, Murray T, Xu J, Thun MJ. Cancer statistics. CA Cancer J Clin 2007; 57: 43-66.

3 Bentrem DJ, Dematteo RP, Blumgart LH. Surgical therapy for metastatic disease to the liver. Annu Rev Med 2005; 56: 139-156. 
4 Mayer-Kuckuk P, Banerjee D, Kemeny N, Fong Y, Bertino JR. Molecular therapies for colorectal cancer metastatic to the liver. Mol Ther 2002; 5: 492-500.

5 Douillard JY, Cunningham D, Roth AD, Navarro M, James RD, Karasek $\mathrm{P}$ et al. Irinotecan combined with fluorouracil compared with fluorouracil alone as first-line treatment for metastatic colorectal cancer: a multicentre randomised trial. Lancet 2000; 355: 1041-1047.

6 Kohne $\mathrm{CH}$, van Cutsem E, Wils J, Bokemeyer C, El-Serafi M, Lutz MP et al. European Organisation for Research and Treatment of Cancer Gastrointestinal Group. Phase III study of weekly high-dose infusional fluorouracil plus folinic acid with or without irinotecan in patients with metastatic colorectal cancer: European Organisation for Research and Treatment of Cancer Gastrointestinal Group Study 40986. J Clin Oncol 2005; 23: $4856-4865$.

7 de Gramont A, Figer A, Seymour M, Homerin M, Hmissi A, Cassidy $\mathrm{J}$ et al. Leucovorin and fluorouracil with or without oxaliplatin as first-line treatment in advanced colorectal cancer. J Clin Oncol 2000; 18: 2938-2947.

8 Grothey A, Sargent D, Goldberg RM, Schmoll HJ. Survival of patients with advanced colorectal cancer improves with the availability of fluorouracil-leucovorin, irinotecan, and oxaliplatin in the course of treatment. J Clin Oncol 2004; 22: 1209-1214.

9 Longley DB, Harkin DP, Johnston PG. 5-Fluorouracil: mechanisms of action and clinical strategies. Nat Rev Cancer 2003; 3: 330-338.

10 Harvey BG, Maroni J, O’Donoghue KA, Chu KW, Muscat JC, Pippo AL et al. Safety of local delivery of low- and intermediatedose adenovirus gene transfer vectors to individuals with a spectrum of morbid conditions. Hum Gene Ther 2002; 13: 15-63.

11 Gene therapy clinical trials world wide. J Gene Med. Available from: www.wiley.co.uk/genmed/clinical.

12 Kievit E, Bershad E, Ng E, Sethna P, Dev I, Lawrence TS et al. Superiority of yeast over bacterial cytosine deaminase for enzyme/prodrug gene therapy in colon cancer xenografts. Cancer Res 1999; 59: 1417-1421.

13 Hamstra DA, Rice DJ, Fahmy S, Ross BD, Rehemtulla A. Enzyme/prodrug therapy for head and neck cancer using a catalytically superior cytosine deaminase. Hum Gene Ther 1999; 10: 1993-2003.

14 Tiraby M, Cazaux C, Baron M, Drocourt D, Reynes JP, Tiraby G. Concomitant expression of $E$. coli cytosine deaminase and uracil phosphoribosyltransferase improves the cytotoxicity of 5-fluorocytosine. FEMS Microbiol Lett 1998; 167: 41-49.

15 Erbs P, Regulier E, Kintz J, Leroy P, Poitevin Y, Exinger F et al. qIn vivo cancer gene therapy by adenovirus-mediated transfer of a bifunctional yeast cytosine deaminase/uracil phosphoribosyltransferase fusion gene. Cancer Res 2000; 60: 3813-3822.

16 Erbs P, Findeli A, Kintz J, Cordier P, Hoffmann C, Geist M et al. Modified vaccinia virus Ankara as a vector for suicide gene therapy. Cancer Gene Ther 2008; 15: 18-28.

17 Ring CJ. Cytolytic viruses as potential anti-cancer agents. J Gen Virol 2002; 83: 491-502.

18 Kirn DH, McCormick F. Replicating viruses as selective cancer therapeutics. Mol Med Today 1996; 2: 519-527.

19 Martuza RL. Conditionally replicating herpes vectors for cancer therapy. J Clin Invest 2000; 105: 841-846.

20 Figova K, Hrabeta J, Eckschlager T. Reovirus-possible therapy of cancer. Neoplasma 2006; 53: 457-462.

21 Shen Y, Nemunaitis J. Fighting cancer with vaccinia virus: teaching new tricks to an old dog. Mol Ther 2005; 11: 180-195.

22 Reid T, Galanis E, Abbruzzese J, Sze D, Wein LM, Andrews J et al. Hepatic arterial infusion of a replication-selective oncolytic adenovirus (d11520): phase II viral, immunologic, and clinical endpoints. Cancer Res 2002; 62: 6070-6079.

23 Sze DY, Freeman SM, Slonim SM, Samuels SL, Andrews JC, Hicks $\mathrm{M}$ et al. Dr Gary J Becker Young Investigator Award: intraarterial adenovirus for metastatic gastrointestinal cancer: activity, radiographic response, and survival. J Vasc Interv Radiol 2003; 14: 279-290.

24 Buller RM, Smith GL, Cremer K, Notkins AL, Moss B. Decreased virulence of recombinant vaccinia virus expression vectors is associated with a thymidine kinase-negative phenotype. Nature 1985; 317: 813-815.

25 Acres B, Dott K, Stefani L, Kieny MP. Directed cytokine expression in tumour cells in vivo using recombinant vaccinia virus. Ther Immunol 1994; 1: 17-23.

26 Puhlmann M, Gnant M, Brown CK, Alexander Jr HR, Bartlett DL. Thymidine kinase-deleted vaccinia virus expressing purine nucleoside phosphorylase as a vector for tumor-directed gene therapy. Hum Gene Ther 1999; 10: 649-657.

27 Puhlmann M, Brown CK, Gnant M, Huang J, Libutti SK, Alexander Jr HR et al. Vaccinia as a vector for tumor-directed gene therapy: biodistribution of a thymidine kinase-deleted mutant. Cancer Gene Ther 2000; 7: 66-73.

28 Taylor G, Stott EJ, Wertz G, Ball A. Comparison of the virulence of wild-type thymidine kinase (tk)-deficient and tk+ phenotypes of vaccinia virus recombinants after intranasal inoculation of mice. J Gen Virol 1991; 72: 125-130.

29 Merchlinsky M, Moss B. Introduction of foreign DNA into the vaccinia virus genome by in vitro ligation: recombinationindependent selectable cloning vectors. Virology 1992; 190: 522-526.

30 Hung CF, Tsai YC, He L, Coukos G, Fodor I, Qin L et al. Vaccinia virus preferentially infects and controls human and murine ovarian tumors in mice. Gene Therapy 2007; 14: 20-29.

31 Gnant MF, Noll LA, Irvine KR, Puhlmann M, Terrill RE, Alexander Jr HR et al. Tumor-specific gene delivery using recombinant vaccinia virus in a rabbit model of liver metastases. J Natl Cancer Inst 1999; 91: 1744-1750.

32 Mesnil M, Piccoli C, Tiraby G, Willecke K, Yamasaki H. Bystander killing of cancer cells by herpes simplex virus thymidine kinase gene is mediated by connexins. Proc Natl Acad Sci USA 1996; 93: 1831-1835.

33 Matono S, Tanaka T, Sueyoshi S, Yamana H, Fujita H, Shirouzu K. Bystander effect in suicide gene therapy is directly proportional to the degree of gap junctional intercellular communication in esophageal cancer. Int J Oncol 2003; 23: 1309-1315.

34 McCart JA, Puhlmann M, Lee J, Hu Y, Libutti SK, Alexander Jr HR et al. Complex interactions between the replicating oncolytic effect and the enzyme/prodrug effect of vaccinia-mediated tumor regression. Gene Therapy 2000; 7: 1217-1223.

35 Nakamura H, Mullen JT, Chandrasekhar S, Pawlik TM, Yoon SS, Tanabe KK. Multimodality therapy with a replication-conditional herpes simplex virus 1 mutant that expresses yeast cytosine deaminase for intratumoral conversion of 5-fluorocytosine to 5-fluorouracil. Cancer Res 2001; 61: 5447-5452.

36 Bernt KM, Steinwaerder DS, Ni S, Li ZY, Roffler SR, Lieber A. Enzyme-activated prodrug therapy enhances tumorspecific replication of adenovirus vectors. Cancer Res 2002; 62: 6089-6098.

37 Schepelmann S, Ogilvie LM, Hedley D, Friedlos F, Martin J, Scanlon I et al. Suicide gene therapy of human colon carcinoma xenografts using an armed oncolytic adenovirus expressing carboxypeptidase G2. Cancer Res 2007; 67: 4949-4955.

38 Eisenberg DP, Adusumilli PS, Hendershott KJ, Yu Z, Mullerad M, Chan MK et al. 5-Fluorouracil and gemcitabine potentiate the efficacy of oncolytic herpes viral gene therapy in the treatment of pancreatic cancer. J Gastrointest Surg 2005; 9: 1068-1077.

39 Karupiah G, Coupar B, Ramshaw I, Boyle D, Blanden R, Andrew M. Vaccinia virus-mediated damage of murine ovaries and protection by virus-expressed interleukin-2. Immunol Cell Biol 1990; 68: 325-333. 
40 Gnant MF, Puhlmann M, Bartlett DL, Alexander Jr HR. Regional versus systemic delivery of recombinant vaccinia virus as suicide gene therapy for murine liver metastases. Ann Surg 1999; 230: 352-360.

41 Naik AM, Chalikonda S, McCart JA, Xu H, Guo ZS, Langham G et al. Intravenous and isolated limb perfusion delivery of wild type and a tumor-selective replicating mutant vaccinia virus in nonhuman primates. Hum Gene Ther 2006; 17: 31-45.

42 Neeman M, Abramovitch R, Schiffenbauer YS, Tempel C. Regulation of angiogenesis by hypoxic stress: from solid tumours to the ovarian follicle. Int J Exp Pathol 1997; 78: $57-70$.

43 Tartaglia J, Perkus ME, Taylor J, Norton EK, Audonnet JC, Cox WI et al. NYVAC: a highly attenuated strain of vaccinia virus. Virology 1992; 188: 217-232.

44 Kretzschmar M, Wallinga J, Teunis P, Xing S, Mikolajczyk R. Frequency of adverse events after vaccination with different vaccinia strains. PLoS Med 2006; 3: 1341-1351.
45 Kieny MP, Lathe R, Drillien R, Spehner D, Skory S, Schmitt D et al. Expression of rabies virus glycoprotein from a recombinant vaccinia virus. Nature 1984; 312: 163-166.

46 Paoletti E. Applications of pox virus vectors to vaccination: an update. Proc Natl Acad Sci USA 1996; 93: 11349-11353.

47 Smith DB, Johnson KS. Single-step purification of polypeptides expressed in Escherichia coli as fusions with glutathione S-transferase. Gene 1988; 67: 31-40.

48 Greene GL, Fitch FW, Jensen EV. Monoclonal antibodies to estrophilin: probes for the study of estrogen receptors. Proc Natl Acad Sci USA 1980; 77: 157-161.

49 McBee P, Petraiuolo W, Katoh A. Inhibition of liver metastases in nude mice by the combined action of 5-fluorouracil and interferon. Anticancer Drugs 1990; 1: 165-170.

50 Sova P, Ren XW, Ni S, Bernt KM, Mi J, Kiviat N et al. A tumortargeted and conditionally replicating oncolytic adenovirus vector expressing TRAIL for treatment of liver metastases. Mol Ther 2004; 9: 496-509. 